\title{
Zu den Auswirkungen der Rangposition auf die Fruchtbarkeits- leistung von Sauen
}

\begin{abstract}
Title of the paper: Impact of rank position on fertility of sows

The investigations were carried out with 460 sows from two farms (farm A: housing the sows in small groups of 8 animals each, farm B with a large group of 100 sows) and a total number of 901 matings. The number of agonistic interactions was registered for each sow during $48 \mathrm{~h}$ after mixing soon after weaning the piglets. The individual rank place in the social hierarchy was calculated on the basis of wins and defeats and the sows were divided in high and low ranking sows. At farm B the rank position was estimated on the basis of the daily feeding order at two electronic feeding stations (first half = high ranking, second half of the sows in the feeding order = low ranking). Additionally, the following parameters were recorded for each sow: parity, farrowing rate and litter size (total and alive born piglets). The analysis showed that sows with a high rank position had a significantly higher farrowing rate (89.8\%) compared to pen-mates with low rank places $(83.9 \%, \mathrm{p}<0.05)($ farm A). Sows with a high rank position reached a significantly higher litter size of total born piglets $(12.51,16.14$ piglets per litter respectively) than the low-ranking pen-mates (12.00, 14.83 piglets/litter respectively - farms A and B). In tendency the difference concerning the litter size between sows with high and low rank position was larger in sows with parity 2 than in sows with a higher parity number (farm A). When mixing sows, the time and the conditions (e.g. group size, space allowance per sow) have to be considered to prevent the negative influence of (low) rank order on fertility.
\end{abstract}

Keywords: sow, social behaviour, rank position, fertility

\section{Zusammenfassung}

Die Untersuchungen wurden an 460 Sauen aus zwei Betrieben mit insgesamt 901 Belegungen durchgeführt, wobei in Betrieb A Kleingruppen (8 Sauen/Gruppe) und in Betrieb B eine Großgruppe mit 100 Sauen analysiert wurden. Beim Gruppieren von 8 Sauen unmittelbar nach dem Absetzen der Ferkel (Betrieb A) wurde über 48 Stunden hinweg die Zahl der agonistischen Interaktionen je Sau ermittelt. Daraus wurde der individuelle Rangplatz innerhalb der sozialen Hierarchie und die Zuordnung zu „ranghoch“ und „rangniedrig“ berechnet. Im Betrieb B wurde die Rangposition auf der Basis der Reihenfolge der Futteraufnahme an zwei elektronischen Futterstationen (erste Hälfte = ranghohe, zweite Hälfte = rangniedere Sauen) bestimmt. Die Angaben zu Parität, Abferkelrate sowie Wurfgröße gesamt und lebend geborener Ferkel ergänzten die Datenerfassung. Die Auswertungen ergaben, dass ranghohe Sauen mit $89,8 \%$ eine signifikant höhere Abferkelrate als rangniedere Tiere $(83,9 \%, p<0,05)$ hatten (Betrieb A). Ranghohe Sauen erzielten eine signifikant höhere Wurfgröße gesamt geborener Ferkel (12,51 bzw. 16,14 Ferkel/Wurf) als ihre rangniederen Buchtengefährtinnen (12,00 bzw. 14,83 Ferkel/Wurf - Betriebe A und B). Bei den Sauen mit Wurfnummer $=2$ war der diesbezügliche Unterschied zwischen ranghohen und rangniederen Sauen etwas deutlicher ausgeprägt als bei den älteren Sauen (Wurfnummer $>2$; Betrieb A). Bei der Gruppenbildung von Sauen sind der Zeitpunkt sowie die Bedingungen (z. B. Gruppengröße, Fläche je Tier) zu berücksichtigen, um rangordnungsbedingte Auswirkungen auf die Fruchtbarkeitsleistung zu begrenzen.

Schlüsselwörter: Sau, Sozialverhalten, Rangposition, Fruchtbarkeit

\section{Einleitung}

Bei der Gruppenbildung von Sauen treten über einen Zeitraum von etwa zwei Tagen teleonome agonistische Interaktionen auf, in deren Folge eine soziale Hierarchie aufgebaut wird (AREY und EDWARDS, 1998; BAUER und HOY, 2002; HOY und 
BAUER, 2005; BORBERG und HOY, 2007). Die mit den Rangordnungskämpfen einhergehende Belastung der Tiere kann zur Beeinträchtigung der Fruchtbarkeitsleistung führen (SCHNURRBUSCH und HÜHN, 1994), wenngleich bislang nur wenige experimentell untersetzte Resultate für diesen Sachverhalt vorliegen. Die Gruppenbildung im Zeitraum der Nidation (zweite bis dritte Graviditätswoche) kann zu einer höheren Umrauscherrate und einer geringeren Wurfgröße führen (TE BRAKE und BRESSERS, 1990; RAZDAN et al., 2004). Ähnliche Ergebnisse ermittelten auch BOKMA (1990) und SIMMINS (1993). In diesen Untersuchungen wurde jedoch nicht zwischen rangniederen und ranghohen Tieren unterschieden. Die vorliegenden Ergebnisse zum möglichen Zusammenhang zwischen Rangposition und Fruchtbarkeitsleistung sind spärlich und widersprüchlich (BAUER, 2005; JARVIS et al., 2006; KRANENDONK et al., 2007; BORBERG, 2008). Somit bestand das Ziel der vorliegenden Untersuchungen darin, die Auswirkungen der Rangposition auf die Fruchtbarkeit einer größeren Zahl von Sauen in zwei Betrieben mit Klein- oder Großgruppenhaltung zu quantifizieren.

\section{Untersuchungsbedingungen und Methoden}

Die Untersuchungen fanden in zwei Betrieben (Betrieb A: 90 Sauen, zumeist Landrasse und Edelschwein sowie Hybriden, Kleingruppenhaltung zu 8 Sauen und Fütterung an Trockenautomaten während der Gruppierung - Betrieb B: 200 Sauen, Danzucht, Großgruppenhaltung mit etwa 100 Sauen und Fütterung an zwei elektronischen Abrufstationen) statt. Die Haltungsbedingungen im Betrieb A wurden ausführlich von BAUER und HOY (2002) sowie HOY et al. (2005), die im Betrieb B von HOY et al. (2007) beschrieben. Die Sauenherde in Betrieb A wird im geschlossenen System in einem Zwei-Wochen-Rhythmus bewirtschaftet. Jeden zweiten Donnerstag werden in der Regel die Ferkel von acht Sauen abgesetzt, worauf die Sauen in eine Stimulations-Bucht kommen (BAUER und HOY, 2005). Die Sauen bleiben 48 Stunden in dieser Stimu-Bucht, da nachgewiesen wurde, dass danach über $90 \%$ aller Rangkämpfe beendet sind (BAUER und HOY, 2002). Anschließend werden die Sauen in das Besamungszentrum in Einzelstände umgestallt und duldungsorientiert besamt. In den vorliegenden Untersuchungen kamen dieselben Sauen nach 7 oder 28 Tagen erneut zur zweiten Gruppierung in die Stimu-Bucht, in der sie wiederum 48 Stunden verblieben. Daran schloss sich die Gruppenhaltung auf einer eingestreuten Lauffläche im Wartestall mit Fressständen an.

Sämtliche agonistischen Interaktionen jeder Sau gegen jede andere während der Gruppierungsphase wurden in einer $8 \times 8$-Matrix erfasst (BAUER und HOY, 2002). Aus der Anzahl der Siege und Niederlagen wurde für jedes Tier der individuelle Rangplatz bestimmt (PUPPE, 1996; LANGBEIN und PUPPE, 2004; PUPPE et al., 2008). Für die weitere Berechnung wurden Tiere mit den Rangplätzen 1 bis 4 (ranghohe Sauen) und 5 bis 8 (rangniedere Tiere) zusammengefasst. Für jede einzelne Sau wurden darüber hinaus folgende Daten zur Fruchtbarkeitsleistung erhoben: Parität, Trächtigkeitsdauer, Abferkelrate (Anzahl abgeferkelter Sauen bezogen auf die erstbesamten Tiere) sowie Wurfgröße gesamt und lebend geborener Ferkel. Es konnten die Werte von 284 Sauen mit 725 Belegungen ausgewertet werden.

In großen Sauengruppen mit 100 Tieren oder mehr ist es aus technischen Gründen schwierig, die Beziehungen der Individuen untereinander aufzuklären. Weder in visuellen Direktbeobachtungen noch durch die Anwendung von Videotechnik ist es möglich, exakt die agonistischen Interaktionen in einer Matrix zu erfassen (BAUER 
und HOY, 2002; HOY et al., 2005), um daraus die soziale Stellung des Einzeltieres bestimmen zu können. Als Hilfsmittel zur indirekten Bestimmung der Rangordnung kann die Reihenfolge der Futteraufnahme an einer elektronischen Abrufstation herangezogen werden (RITTER und WEBER, 1988; JENSEN et al., 2000). Ranghöhere Sauen rufen vor den rangniederen Tieren ihre Futterration ab (HOY et al., 2007). Die Untersuchungen im Betrieb B fanden in einem Außenklimastall mit Einstreu im Liegebereich und kompaktem Betonboden im Laufbereich statt. Eine Großbucht mit zwei elektronischen Abrufstationen (EFS) bot Platz für 100 Sauen. Die beiden Abrufstationen besaßen die Voraussetzung, automatisch die Reihenfolge der Stationsbesuche der Sauen mit Futterabruf in Zuordnung zur Realzeit zu erfassen. In den Wartestall wurden im dreiwöchigen Rhythmus tragend getestete Sauen als Untergruppe in die Großgruppe eingegliedert.

Im Untersuchungszeitraum, der sich über ein Jahr erstreckte, konnte insgesamt zehnmal die Eingliederung neuer Untergruppen $(n=16$ bis 24) in die dynamische Großgruppe, die dann zwischen 77 und 98 Sauen umfasste, untersucht werden. Es konnten eine sehr hohe Wiederholbarkeit der Reihenfolge der Futterstationsbesuche an konsekutiven Tagen mit Korrelationskoeffizienten von z. T. über 0,90 und eine hochsignifikante Beziehung zwischen Parität und Reihenfolge des Futterabrufes nachgewiesen werden (HOY et al., 2007). Die Sauen konnten an beiden Stationen Futter abrufen, wobei im Untersuchungszeitraum gleichmäßig verteilt bis zu 50 Sauen pro Station gefüttert wurden. Sauen in der ersten Hälfte der Besuchsreihenfolge an der Futterstation (Platzziffern 1 bis 25) wurden als ranghohe Sauen und die anderen Gruppengefährtinnen (Platzziffer 26 bis 50) als rangniedere Tiere definiert. Insgesamt konnten 176 Sauen (ausschließlich Altsauen) in die Untersuchungen einbezogen werden.

Sämtliche Einzeltierdaten wurden mit Hilfe des Programmpaketes SPSS 12.0 for Windows biostatistisch analysiert. Zur Prüfung der Häufigkeitsunterschiede (Abferkelrate) kam der Chi-Quadrat-Unabhängigkeitstest in Kontingenztafeln zur Anwendung. Die Mittelwertdifferenzen (Wurfgröße) zwischen rangniederen und ranghohen Sauen wurden mittels univariater Varianzanalyse und folgendem Modell (Betrieb A) auf Signifikanz geprüft:

$$
\mathrm{Y}=\mu+\text { Rangstatus }_{\mathrm{i}}+\text { Wurfnummer }_{\mathrm{j}}+\text { Genotyp }_{\mathrm{k}}+\mathrm{e}_{\mathrm{ijk}}
$$

mit Rangstatus (niedrig, hoch), Wurfnummer und Genotyp als fixe Effekte $(p<0,05)$. Da alle Sauen im Betrieb B derselben Genetik angehörten, wurden in das entsprechende statistische Modell lediglich Rangstatus und Parität als fixe Effekte und das Alter in Tagen als Kovariable aufgenommen.

\section{Ergebnisse}

Insgesamt konnten im Betrieb A 725 Belegungen bei 284 Sauen und daraus 630 erbrachte Würfe hinsichtlich der Wurfgröße gesamt und lebend geborener Ferkel ausgewertet werden. Aus den 371 Besamungen ranghoher Sauen resultierte eine Abferkelrate von $89,8 \%$, bei den rangniederen Tieren betrug der Anteil abferkelnder Sauen 83,9\%. Die Differenz in Höhe von 5,9\% zwischen Sauen mit hoher und niedriger Rangposition war signifikant $(\mathrm{p}<0,05)$ (Abbildung 1).

In der Trächtigkeitsdauer bestand kein signifikanter Unterschied zwischen Tieren mit differenter Rangposition. Ranghohe Sauen hatten eine mittlere Graviditätsdauer von 114,4 Tagen, ihre rangniederen Gruppengefährtinnen besaßen einen Wert von 114,9 Tagen. 


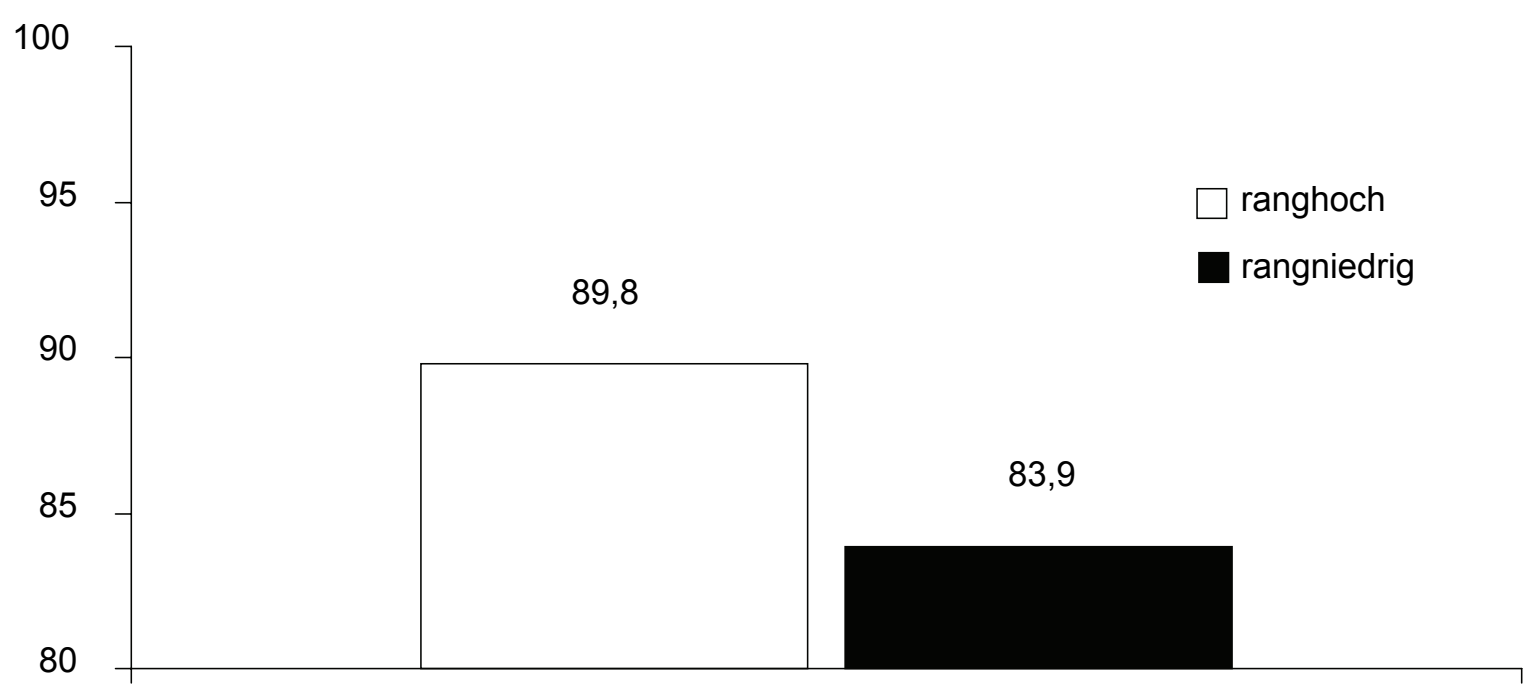

Abb. 1: Abferkelrate bei Sauen mit hoher bzw. niedriger Rangposition (Farrowing rate depending on rank position)

Die Ergebnisse der univariaten Varianzanalyse wiesen einen signifikanten Einfluss der Rangposition der Sauen auf ihre Fruchtbarkeitsleistung nach. Ranghohe Sauen erreichten eine signifikant höhere Wurfgröße gesamt geborener Ferkel als ihre rangniederen Buchtengefährtinnen (Tabelle 1). Bei der Wurfgröße gesamt geborener Ferkel betrug der Leistungsvorteil der Tiere mit hoher Rangposition 0,51 Ferkel im Mittel von insgesamt 630 Abferkelungen. Ranghohe Sauen $(n=333)$ erzielten eine mittlere Wurfgröße gesamt geborener Ferkel von 12,51, die rangniederen Vergleichssauen $(n=297)$ einen Wert von 12,00. Die entsprechenden Werte für die Wurfgröße lebend geborener Ferkel betrugen für ranghohe Sauen 11,53 und für Tiere mit niedriger Rangposition 11,21 ( $>>0,05)$. Somit erzielten die ranghohen Sauen in vorliegenden Untersuchungen bei gleichzeitiger Berücksichtigung der Effekte von Wurfnummer und Rasse 0,32 lebend geborene Ferkel mehr pro Wurf als rangniedere Vergleichstiere.

Tabelle 1

Einfluss der Rangposition auf die Wurfgröße im Folgewurf (Betrieb A)

(Influence of rank position on litter size at the following parturition [farm A])

\begin{tabular}{lcccc}
\hline & Rangposition & $\mathrm{n}$ & Mittelwert & Standardfehler \\
\hline \multirow{2}{*}{ Wurfgröße gesamt geborener Ferkel } & hoch & 333 & $12,51^{\mathrm{a}}$ & 0,16 \\
& niedrig & 297 & $12,00^{\mathrm{a}}$ & 0,19 \\
\multirow{2}{*}{ Wurfgröße lebend geborener Ferkel } & hoch & 333 & 11,53 & 0,16 \\
& niedrig & 297 & 11,21 & 0,18 \\
\hline
\end{tabular}

Gleiche Buchstaben markieren einen signifikanten Unterschied $(\mathrm{p}<0,05)$.

Darüber hinaus wurde die univariate Varianzanalyse mit den fixen Effekten von Rangposition und Genotyp getrennt für Sauen mit der Parität $=2$ (nach dem 2. Wurf) und Sauen mit Parität 3 bis 6 berechnet, da es sich hierbei um die größten Teilstichproben mit 114 bzw. 278 Würfen handelte. In beiden Stichproben ließ sich der Einfluss der Rangposition auf die Wurfgröße wegen der großen Differenz trotz der geringeren Anzahl Probanden statistisch sichern. In den Teilgruppen erzielten die ranghohen Sauen eine um 1,32 bzw. 0,95 gesamt geborene Ferkel höhere Wurfgröße als die rangniederen Sauen (Tabelle 2). 
Tabelle 2

Einfluss der Rangposition auf die Wurfgröße gesamt geborener Ferkel bei Sauen mit 2 bzw. 3 bis 6 Würfen (Betrieb A) (Influence of rank position on litter size total born piglets in sows with 2, 3 to 6 parities respectively [farm A])

\begin{tabular}{lcccc}
\hline & Rangposition & $\mathrm{n}$ & Mittelwert & Standardfehler \\
\hline Parität $=2$ & hoch & 66 & $13,34^{\mathrm{a}}$ & 0,37 \\
Wurfgröße gesamt geborener Ferkel & niedrig & 48 & $12,02^{\mathrm{a}}$ & 0,54 \\
Parität $=3$ bis 6 & hoch & 178 & $12,96^{\mathrm{b}}$ & 0,21 \\
Wurfgröße lebend geborener Ferkel & niedrig & 100 & $12,01^{\mathrm{b}}$ & 0,33 \\
\hline
\end{tabular}

Jeweils gleiche Buchstaben markieren signifikante Unterschiede $(\mathrm{p}<0,05)$.

Im Betrieb B konnte 176 Sauen eine Rangposition zugewiesen werden: 90 Sauen wurden auf der Basis ihrer Reihenfolge bei der Futteraufnahme in der Abrufstation als ranghoch und 86 zeitgleich aufgestallte Tiere als rangnieder definiert. Unter Berücksichtigung der Parität als fixer Effekt und des Alters in Tagen zum Zeitpunkt der Beobachtung als Kovariable im statistischen Modell konnte analog zum Betrieb A auch bei der Großgruppenhaltung ein Einfluss der Rangposition auf die Wurfgröße gesamt geborener Ferkel nachgewiesen werden. Ranghohe Sauen erzielten im Mittel um 1,31 gesamt und 0,72 lebend geborene Ferkel größere Würfe als die rangniedrigeren Gruppengefährtinnen (Tabelle 3).

Tabelle 3

Einfluss der Rangposition auf die Wurfgröße im Folgewurf (Betrieb B)

(Influence of rank position on litter size at the following parturition [farm B])

\begin{tabular}{lcccc}
\hline & Rangposition & $\mathrm{n}$ & Mittelwert & Standardfehler \\
\hline \multirow{2}{*}{ Wurfgröße gesamt geborener Ferkel } & hoch & 90 & $16,14^{\mathrm{a}}$ & 0,46 \\
& niedrig & 86 & $14,83^{\mathrm{a}}$ & 0,54 \\
\multirow{2}{*}{ Wurfgröße lebend geborener Ferkel } & hoch & 90 & 13,45 & 0,40 \\
& niedrig & 86 & 12,73 & 0,47 \\
\hline
\end{tabular}

Gleiche Buchstaben markieren einen signifikanten Unterschied $(p=0,052)$.

\section{Diskussion}

Nach der EU-Richtlinie 2001/88 und ihrer Umsetzung in nationales Tierschutzrecht durch die Tierschutz-Nutztierhaltungsverordnung müssen spätestens ab 1. Januar 2013 tragende Sauen ab dem 29. Trächtigkeitstag bis eine Woche vor der voraussichtlichen Abferkelung in Gruppen gehalten werden. Jede Gruppenhaltung beginnt mit der Gruppierung, und dabei treten unvermeidliche Rangordnungskämpfe auf, die auch durch die verschiedensten Management-Maßnahmen nicht verhindert, höchstens verzögert werden können (BORBERG und HOY, 2008). Diese agonistischen Interaktionen sind nämlich biologisch zweckmäßig, da hierdurch eine soziale Dominanzhierarchie aufgebaut wird, die ständige Kämpfe weitgehend verhindert (zusammenfassende Literatur bei AREY und EDWARDS, 1998; BAUER und HOY, 2002; HOY et al., 2005). Immer fortdauernde Kämpfe könnten zu Verletzungen führen und sind energetisch aufwändig. Bereits mehrfach wurde darauf hingewiesen, dass die Rangkämpfe Auswirkungen auf die Fruchtbarkeitsleistung haben können (TE BRAKE und BRESSERS, 1990; BOKMA, 1990; SIMMINS, 1993; HOY und LUTTER, 1995). Erklärt wird dies damit, dass verschiedene Stressoren - darunter soziale Stressoren auf die Hypothalamus-Hypophysen-Gonaden-Achse einwirken und zu einer Inhibierung der hypothalamischen GnRH-Sekretion sowie in der Folge davon zu einer reduzierten LH-Freisetzung im Hypophysenvorderlappen führen (SCHNURRBUSCH und HÜHN, 1994; VON BORELL, 2000; MANTEUFFEL, 2002; TUCHSCHERER und 
MANTEUFFEL, 2000; TUCHSCHERER und PUPPE, 2000). ZANELLA et al. (1996) fanden unterschiedliche Cortisol-Spiegel bei Sauen mit differenter Rangposition. Während für den Durchschnitt der Gruppe der Einfluss der Gruppierung im Zusammenhang mit dem Gruppierungszeitpunkt auf Parameter der Fruchtbarkeit vereinzelt nachgewiesen wurde (TE BRAKE und BRESSERS, 1990; BOKMA, 1990; SIMMINS, 1993), sind die Angaben in der Literatur zu den Auswirkungen der Rangposition auf die Leistungsdaten uneinheitlich. Nach JARVIS et al. (2006) und KRANENDONK et al. (2007) hat der soziale Rang der Sauen keinen Einfluss auf die Wurfgröße, jedoch auf die Leistung und das Verhalten der Nachkommen. In den Untersuchungen von BAUER (2005) und BORBERG (2008) deutete sich dagegen eine Auswirkung der Rangposition (ranghoch, rangniedrig) auf die Fruchtbarkeitsleistung an, allerdings reichte der Stichprobenumfang nicht für eine statistische Absicherung aus.

In Gruppen von Sauen gibt es grundsätzlich Tiere mit hoher und solche mit niedriger Rangposition. Mit den vorliegenden Untersuchungen wurde erstmals nachgewiesen, dass zwischen ranghohen und rangniederen Tieren signifikante Unterschiede in der Abferkelrate und in der Wurfgröße gesamt und lebend geborener Ferkel bestehen. In der Zusammenfassung aller Sauen im Betrieb A ( $\mathrm{n}=725$ Belegungen, aus denen 630 Würfe resultierten) erreichten ranghohe Sauen mit $89,8 \%$ eine signifikant höhere Abferkelrate als ihre rangniederen Buchtengefährtinnen (83,9 \%).

In die Untersuchungen waren ausschließlich Altsauen einbezogen worden. Im Brunstverhalten (Eintritt der Brunst bzw. erste Belegung in Tagen nach dem Absetzen der Ferkel), in der Anzahl Belegungen pro Sau und in der Trächtigkeitsdauer bestanden keine Unterschiede zwischen ranghohen und rangniederen Tieren.

Mittels univariater Varianzanalyse ließ sich zeigen, dass der Unterschied in der Wurfgröße gesamt und lebend geborener Ferkel zwischen Sauen mit hoher oder niedriger Rangposition auch dann zwischen 0,5 und 0,7 Ferkel pro Wurf zugunsten der ranghohen Tiere betrug, wenn der Einfluss der fixen Effekte von Wurfnummer und Genotyp berücksichtigt wurde (Betrieb A). Ein gleichgerichtetes Ergebnis trat ebenso im Betrieb B bei Großgruppenhaltung zutage. Die ranghohen Sauen hatten 1,3 gesamt geborene und 0,7 lebend geborene Ferkel pro Wurf mehr als rangniedere Gruppengefährtinnen. Die Differenz bei der Wurfgröße gesamt geborener Ferkel lag an der Signifikanzschwelle $(\mathrm{p}=0,052)$.

Vor diesem Hintergrund sollte im Betrieb A geprüft werden, ob die jüngeren Sauen stärkere Auswirkungen auf die Fruchtbarkeitsleistung hinnehmen mussten und ob bei älteren Sauen der Unterschied zwischen rangniederen und ranghöheren Gruppengefährtinnen bezüglich leistungsdepressiver Effekte eventuell geringer ist. Es zeigte sich tatsächlich, dass bei Sauen mit Parität $=2$ der Unterschied in der Wurfgröße zwischen Tieren in der oberen und unteren Hälfte der Ranghierarchie mit 1,3 gesamt geborenen Ferkeln/Wurf größer war als bei den älteren Sauen (Wurfnummern 3 bis 6) mit 0,95 (beide Differenzen signifikant mit $\mathrm{p}<0,05$ ). In der Gruppe der Sauen mit Wurfnummer 3 bis 6 waren mehr ranghohe als rangniedere Sauen vertreten, da in dieser Alterskategorie generell mehr dominante Tiere (im Vergleich zu den jüngeren Sauen) auftreten.

Nach den vorgestellten Ergebnissen muss berücksichtigt werden, dass bei der Gruppenzusammenstellung vor allem bei den rangniederen Sauen Auswirkungen auf die Fruchtbarkeitsleistung (Prozentsatz von Tieren, die nicht tragend werden bzw. umrauschen; Wurfgröße) auftreten können, und zwar in großen und in kleinen Gruppen. Um tierschutzrelevante Schmerzen, Leiden und Schäden (Verlust einzelner 
Embryonen/Feten oder der gesamten Gravidität) bei den Sauen zu verhindern, müssen bei der Gruppenbildung die Anforderungen sowohl der ranghohen als auch der rangniederen Sauen hinsichtlich Ort, Zeit und Bedingungen berücksichtigt werden (BAUER, 2005; BORBERG und HOY, 2007, 2008).

\author{
Literatur \\ AREY, D.S.; EDWARDS, S.A.: \\ Factors influencing aggression between sows after mixing and the consequences for welfare and \\ production. Livestock Prod. Sci. 56 (1998), 61-70
}

BAUER, J.:

Untersuchungen zur Gruppenbildung von Sauen unter Verhaltens-, Gesundheits- und Leistungsaspekten. Diss. Univ. Gießen (2005)

BAUER, J.; HOY, ST.:

Zur Häufigkeit von Rangordnungskämpfen beim ersten und wiederholten Zusammentreffen von Sauen zur Gruppenbildung. Proc. Aktuelle Arbeiten zur artgemäßen Tierhaltung. KTBL-Schrift 418 (2002), $181-187$

BAUER, J.; HOY, ST.:

Die Stimubucht - ein neues Verfahren zur Gruppenbildung von Sauen. Proc. 7. Tagung Bau, Technik

BOKMA, S.: und Umwelt. Braunschweig 01.-03.03.2005, 433-438

BORBERG, A.:

Housing and management of dry sows in groups in practice: partly slatted systems. Proc. Intern. Symp. Electronic Identification in Pig Production. RASE. Stoneleigh (1990), 37-45

Analyse der agonistischen Interaktionen bei der Gruppierung von Sauen mit oder ohne Eber. Diss. Univ. Gießen (2008)

BORBERG, A., HOY, ST.:

Gruppenbildung mit oder ohne Präsenz eines Ebers. Proc. Aktuelle Arbeiten zur artgemäßen Tierhaltung, KTBL-Schrift 461 (2007), 194-202

BORBERG, A., HOY, ST.:

Gruppenhaltung beginnt mit Gruppenbildung. Proc. 13. Inter. Fachtagung Nürtingen 21.-22.02.2008, 27-38

BORELL VON, E.:

Stress and coping in farm animals. Arch. Tierz. 43 (2000) Sonderheft, 144-152

HOY, ST.; LUTTER, C.:

Einfluss der Haltung der Sauen auf den Geburtsverlauf und die Vitalität der Ferkel. Tierärztl. Praxis 23 (1995), 367-372

HOY, ST.; BAUER, J.:

Dominance relationships between sows dependent on the time interval between separation and reunion. Appl. Anim. Behav. Sci. 90 (2005), 21-30

HOY, ST.; BAUER, J.; WEIRICH, C.:

Soziometrische Untersuchungen bei der Gruppenbildung von Sauen. Proc. Aktuelle Arbeiten zur artgemäßen Tierhaltung. KTBL-Schrift 437 (2005), 173-185

HOY, ST.; WEIRICH, C., KRAUSS, V.:

Untersuchungen zum Sozialverhalten von Sauen an elektronischen Abrufstationen. Proc. Aktuelle Arbeiten zur artgemäßen Tierhaltung. KTBL-Schrift 461 (2007), 186-193

JARVIS, S.; MOINARD, S.; ROBSON, S.K.; BAXTER, E.; ORMANDY, E.; DOUGLAS, J.R.; SECKL, J.A.;

RUSSELL, J.A.; LAWRENCE, A.B.:

Programming the offspring of the pig by prenatal stress: Neuroendocrine activity and behaviour. Horm. Behav. 49 (2006), 68-80

JENSEN, K.H.; SORENSEN, L.S.; BERTELSEN, D.; PEDERSEN, E.; JORGENSEN, E; NIELSEN, N.P.; VESTERGARD, K.S.:

Management factors affecting activity and aggression in dynamic group housing systems with electronic sow feeding: a field trial. Anim. Sci. 76 (2000), 535-545

KRANENDONK, G.; VAN DER MHEEN. H.; FILLERUP, M.; KOPSTER, H.:

Social rank of pregnant sows affects their body weight gain and behavior and performance of the offspring. J. Anim. Sci. 85 (2007), 420-429

LANGBEIN, J.; PUPPE, B.:

Analysing dominance relationships by sociometric methods - a plea for a more standardised and precise approach in farm animals. Appl. Anim. Behav. Sci. 87 (2004), 293-315

MANTEUFFEL, G.:

Central nervous regulation of the hypothalamic-pituitary-adrenal axis and ist impact on fertility, immunity, metabolism and animal welfare - a review. Arch. Tierz. 45 (2002), 575-595 
PUPPE, B.:

Soziale Dominanz- und Rangbeziehung beim Hausschwein: eine kritische Übersicht. Berl. Münch. Tierärztl. Wschr. 109 (1996), 457-464

PUPPE, B.; LANGBEIN, J.; BAUER, J.; HOY, ST.:

A comparative view on social hierarchy formation at different stages of pig production using sociometric measures. Livestock Sci. 113 (2008), 155-162

RAZDAN, P.; TUMMARUK, P.; KINDAHL, H.; RODRIGUEZ-MARTINEZ, H.; HULTEN, F.; EINARSSON, S.: Hormonal profiles and embryo survival of sows subjected to induced stress during days 13 and 14 of pregnancy. Anim. Reprod. Sci. 81 (2004), 295-312

RITTER, E; WEBER, R.:

Soziale Rangordnung von Zuchtsauen und Belegung der Futterstation bei zwei verschiedenen Abrufanlagen. Proc. Aktuelle Arbeiten zur artgemäßen Tierhaltung. KTBL-Schrift 336 (1988), 132-141

SCHNURRBUSCH, U.; HÜHN, U.:

Fortpflanzungssteuerung beim weiblichen Schwein. Vetspezial. Verlag Gustav Fischer Jena (1994), 50

SIMMINS, P.H.:

Reproductive performance of sows entering stable and dynamic groups after mating. Anim. Prod. 57 (1993), 293-298

TE BRAKE, J.H.A.; BRESSERS, H.P.M.:

Applications in service management and oestrus detection. Proc. Intern. Symp. Electronic Identification in Pig Production. RASE. Stoneleigh (1990), 63-67

TUCHSCHERER, M.; MANTEUFFEL, G.:

Die Wirkung von psychischem Stress auf das Immunsystem. Ein weiterer Grund für tiergerechte Haltung. Arch. Tierz. 43 (2000) 547-560

TUCHSCHERER, M.; PUPPE, B.:

Dominance status affects immune response after social disturbance in pigs. Arch. Tierz. 43 (2000) Sonderheft, 227

ZANELLA, A.J.; BRUNNER, P.; UNSHELM, J.; MENDL, M.; BROOM, D.M.:

Der Einfluss von Haltungssystem und sozialer Rangordnung auf die Sekretion von Cortisol, $\beta$ Endorphin und Dynorphin bei Sauen. Proc. Aktuelle Arbeiten zur artgemäßen Tierhaltung, KTBLSchrift 373 (1996), 15-25

Eingegangen: 2008-04-11

Akzeptiert: 2008-06-25

Autoren:

Prof. Dr. STEFFEN HOY*

Dr. JÖRG BAUER

Dr. ANNA CATRIN BORBERG

CARMEN WEIRICH

Justus-Liebig-Universität Gießen

Institut für Tierzucht und Haustiergenetik

Bismarckstraße 16

35390 Gießen

Deutschland/Germany

*korrespondierender Autor

E-Mail: steffen.hoy@agrar.uni-giessen.de 